\title{
THE DEPENDENCE OF GALAXY MORPHOLOGY AND STRUCTURE ON ENVIRONMENT AND STELLAR MASS
}

\author{
ARJEN VAN DER WeL \\ Department of Physics and Astronomy, Johns Hopkins University, 3400 North Charles Street, Baltimore, MD 21218; wel@pha.jhu.edu \\ Received 2007 October 31; accepted 2008 January 12; published 2008 January 25
}

\section{ABSTRACT}

From the Sloan Digital Sky Survey (SDSS) Data Release 5 (DR5), we extract a sample of 4594 galaxies at redshifts $0.02<z<0.03$, complete down to a stellar mass of $M=10^{10} M_{\odot}$. We quantify their structure (Sérsic index), morphology (Sérsic index + "Bumpiness"), and local environment. We show that morphology and structure are intrinsically different galaxy properties, and we demonstrate that this is a physically relevant distinction by showing that these properties depend differently on galaxy mass and environment. Structure mainly depends on galaxy mass whereas morphology mainly depends on environment. This is driven by variations in star formation activity, as traced by color, which only weakly affects the structure of a galaxy but strongly affects its morphological appearance. The implication of our results is that the existence of the morphology-density relation is intrinsic and not just due to a combination of more fundamental, underlying relations. Our findings have consequences for high-redshift studies, which often use some measure of structure as a proxy for morphology. A direct comparison with local samples selected through visually classified morphologies may lead to biases in the inferred evolution of the morphological mix of the galaxy population, and misinterpretations in terms of how galaxy evolution depends on mass and environment.

Subject headings: galaxies: fundamental parameters — galaxies: statistics — galaxies: structure

\section{INTRODUCTION}

The morphology-density relation (MDR; Dressler 1980) implies that the environment affects the star formation history, color, and structure of galaxies. Many possible mechanisms have been suggested to explain the suppressed or quenched star formation activity of galaxies located in dense environments, most notably ram pressure stripping (Gunn \& Gott 1972), harassment (Farouki \& Shapiro 1981; Moore et al. 1996), strangulation (e.g., Larson et al. 1980; Kauffmann et al. 1993; Diaferio et al. 2001), and tidal interactions and merging (e.g., Park et al. 2008). At the same time, it is now also well established that strong correlations exist between galaxy mass and, for example, color (e.g., Baldry et al. 2006) and star formation rate (e.g., Brinchmann et al. 2004). Several suggestions have been made to explain the dependence of star formation history on galaxy mass, all of which are related to feedback mechanisms via either supernovae (e.g., White \& Frenk 1991), AGNs (e.g., Croton et al. 2006), or shock heating of infalling gas (Dekel \& Birnboim 2006).

Galaxy structure as measured by, e.g., concentration or Sérsic index, behaves differently from color and star formation in the sense that structure depends strongly on galaxy mass but only weakly on environment (Hogg et al. 2004; Kauffmann et al. 2004). In the context of the MDR this may be surprising as it is clear that concentration and morphology are closely related quantities (e.g., Bell et al. 2003). One possibility is that morphology and concentration only correlate with environment through the underlying correlation between environment and galaxy mass. Another possibility is that morphology and concentration are intrinsically different galaxy properties. For example, it has been suggested (by, e.g., Kauffmann et al. 2004) that morphology is strongly related to star formation, more so than concentration.

Blakeslee et al. (2006) developed an automated, quantitative scheme, the $B-n$ method ( $B$ for "Bumpiness" and $n$ for Sérsic [1968] index; see § 2), to distinguish E+S0 galaxies from later types in Hubble Space Telescope imaging of distant clusters. In van der Wel et al. (2007, hereafter vdW07) we generalize the $B-n$ method to classify galaxies both at high redshift and in the Sloan Digital Sky Survey (SDSS). In both cases the classifications from the $B-n$ method agree very well with visually determined morphologies as long as only two types are considered. In the current Letter we study, for a local sample extracted from the SDSS, the relation between structure (in this Letter measured by Sérsic index $n$ ), morphology (measured by $n$ and $B$ ), color, mass, and environment. This allows us to decide which factors determine the morphological appearance of a galaxy, and provides insight into whether or not the mechanisms that are responsible for morphological transformations are identical to the processes that suppress star formation.

We use the Fifth Data Release (DR5) from the SDSS (Adelman-McCarthy et al. 2007) and adopt the cosmological parameters $\left(\Omega_{M}, \Omega_{\Lambda}, h\right)=(0.3,0.7,0.7)$. The stellar masses used in this Letter are calculated for a "diet" Salpeter (1955) IMF (Bell et al. 2003).

\section{DATA}

We extract a sample of galaxies from the SDSS DR5 at redshifts $0.02<z<0.03$. This is the sample described in vdW07, but extended down to a stellar mass of $M=10^{10}$ $M_{\odot}$ and limited to $z<0.03$. The faintest galaxies in this sample have total magnitudes $r \sim 16.5$ or $g \sim 17.5$, such that spectroscopic completeness is ensured. For details concerning the determination of stellar masses, morphologies, and environment, see vdW07. In short, stellar masses are derived from rest-frame $g-r$ colors, using the empirical calibrations as described by Bell et al. (2003). Local surface densities are estimated by measuring the distance to the seventh nearest neighbor with the same radial velocity within $1000 \mathrm{~km} \mathrm{~s}^{-1}$. The photometric parameters (luminosity and rest-frame color) are derived from the SDSS pipeline archive. The $K$-corrections, to obtain the rest-frame $z=0$ colors $(u-g)_{0}$ and $(g-r)_{0}$, and a correction on the total magnitude to account for a known problem with the SDSS pipeline for bright galaxies, are described in vdW07.

Morphologies are determined by measuring $n$ with GALFIT (Peng et al. 2002) and subsequently the dimensionless param- 


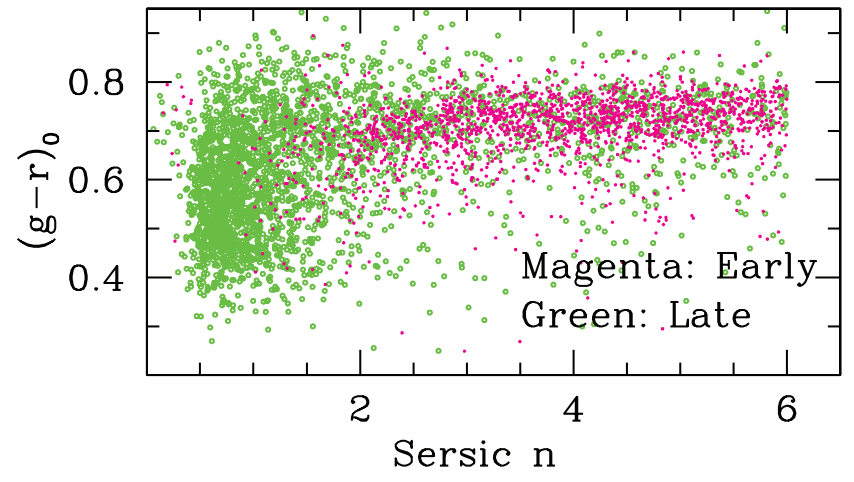

FIG. 1.-Rest-frame $g-r$ color vs. Sérsic index $n$. Magenta points are earlytype $(\mathrm{E}+\mathrm{S} 0)$ galaxies, and green circles are late-type $(\mathrm{Sa}+$ later $)$ galaxies, both according to the $B-n$ method. There are significant number of late-type galaxies with high Sérsic indexes.

eter $B$, which is the rms in the residual divided by the mean of the fit within two effective radii (Blakeslee et al. 2006). To classify galaxies we use the following combination of $B$ and $n$ : galaxies with $B<0.065(n+0.85)$ are considered early types $(\mathrm{E}+\mathrm{S} 0)$, all others late types (Sa and later). This criterion agrees with visually determined morphologies $90 \%$ of the time, and, more importantly for our purposes, the systematic difference in the relative numbers of early- and late-type galaxies is less than 1\% (see Blakeslee et al. 2006 and vdW07). Simulations in which we add noise to the images establish that, with the resolution and depth of SDSS $g$-band imaging, visually determined morphologies can be reliably reproduced with the $B-n$ method down to $g=17.5$, all the way down to the faintest galaxies in our sample.

\section{MORPHOLOGY AND STRUCTURE}

In the literature different observables are used to distinguish different types of galaxies. One can think of morphology, Sérsic index or concentration, and color. In Figure 1 we show that, to first order, there is good correspondence between morphology (according to the $B-n$ method; see $\S 2$ ), structure (Sérsic index), and $(g-r)_{0}$ color, and that the distributions are bimodal for all three. There are red, early-type galaxies with high Sérsic indexes, and blue, late-type galaxies with low Sérsic indexes. However, there are important deviations from this simple picture. For example, there are many red galaxies with low Sérsic indexes, and there are many late-type galaxies with high Sérsic indexes.

In order to quantify the degree of overlap between these galaxy properties, we use three criteria, each of which separates our sample into two subsamples. The first is the morphological classification criterion based on the $B-n$ method. The second criterion is based on the Sérsic index alone (i.e., structure): galaxies with $n>2.5$ are separated from those with $n<2.5$. The third criterion is based on color: galaxies with $(g-$ $r)_{0}>0.077\left[\log \left(M / M_{\odot}\right)-11\right]+0.663$ are considered red, all others blue. This color criterion is based on the least-squares linear fit performed on the early-type galaxies alone (as selected by the $B-n$ method), and iteratively rejecting $3 \sigma$ outliers, where $\sigma$ is the scatter in $(g-r)_{0}$ around the fit (see Fig. 2). We separate blue and red galaxies by shifting the least-squares linear fit down by $2 \sigma=0.085 \mathrm{mag}$.

Down to our mass limit of $10^{10} M_{\odot}$ only $51 \%$ of the red galaxies have early-type morphologies. The red fraction among early-type galaxies, on the other hand, is as high as $90 \%$. Blue, early-type galaxies are rare, and the $10 \%$ found in our sample

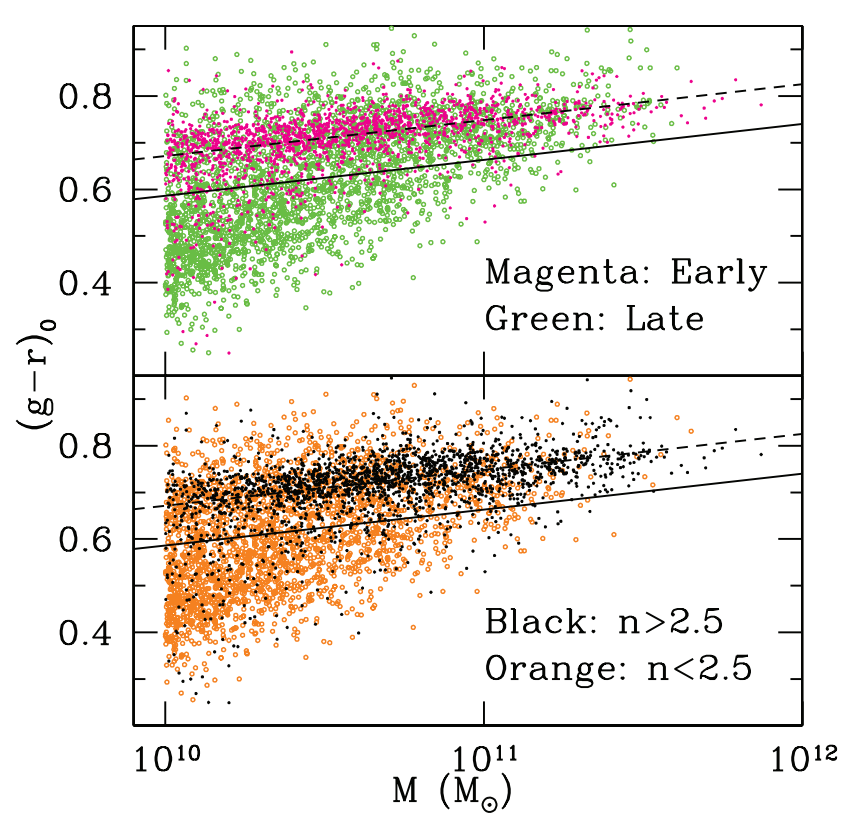

FIG. 2.-Rest-frame $g-r$ color vs. stellar mass. Top: Magenta points are early-type galaxies, and green circles indicate late-type galaxies, both according to the $B$ - $n$ method. Bottom: Black points are galaxies with $n>2.5$, and orange circles are galaxies with $n<2.5$. The dashed lines show the least-squares linear fit to the early-type galaxies, i.e., the magenta points in the top panel, iteratively rejecting $3 \sigma$ outliers. The solid lines, used to separate blue and red galaxies, are the same as the dashed lines but shifted blueward by $2 \sigma$.

could be entirely due to misclassifications, as the random error in the $B-n$ method is $10 \%$ for individual galaxies (see vdW07).

As many as $34 \%$ of the galaxies with $n>2.5$ have late-type morphologies, which may come as a surprise, since usually galaxies with high Sérsic indexes, i.e., de Vaucouleurs profiles, are thought of as bulge-dominated early types. An important aspect of this difference is illustrated in Figure 2: the high- $n$ galaxies (black data points in the bottom panel) have systematically higher masses than the early-type galaxies (magenta data points in the top panel). Apparently, the mass distribution of galaxies with high Sérsic indexes is different from the mass distribution of early-type galaxies.

The different behavior of morphology and structure as a function of mass is illustrated further in the right-hand panels of Figure 3. The bottom right panel shows that the fraction of galaxies with $n>2.5$ depends strongly on galaxy mass. On the other hand, the early-type fraction does not change significantly with increasing mass.

The strong correlation between structure and mass is a reproduction of the result by Kauffmann et al. (2004) even though those authors measure structure differently, by the concentration index $C$, the ratio between the radii containing $90 \%$ and $50 \%$ of the light in the $r$-band. This similarity is no surprise as $C$ and $n$ both depend solely on the change in the slope of the surface brightness profile with radius.

Structure and morphology not only behave differently with respect to galaxy mass, but also with respect to environment: for galaxies with a given mass, the fraction of early types increases with projected surface density (Fig. 3, top left). The dependence of morphology on environment is therefore stronger than the dependence of morphology on mass. For structure this trend is reversed: the fraction of galaxies with $n>2.5$ also modestly increases over the same range in density; however, the dependence of galaxy mass is much stronger (Fig. 3, bottom 


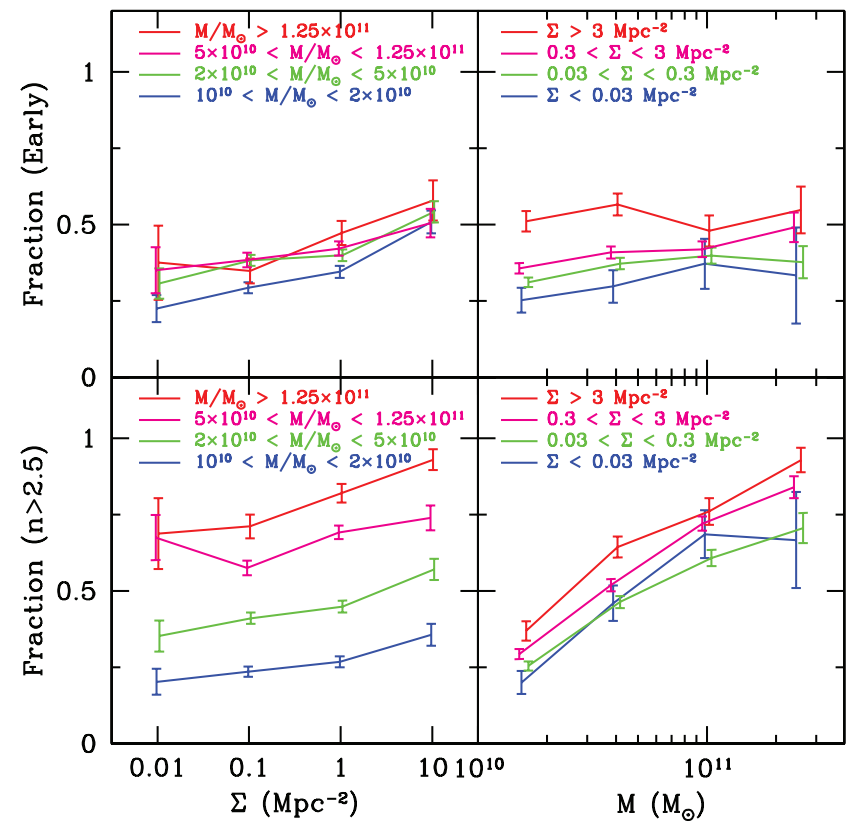

FIG. 3.-Top left: Relation between morphology and environment for galaxies in four different mass bins. Top right: Relation between morphology and galaxy mass for different density bins. Bottom left: Relation between structure (Sérsic parameter $n$ ) and local surface density for galaxies in different mass bins. Bottom right: Relation between structure and galaxy mass in different density bins. Morphology depends mostly on environment, not on galaxy mass. Structure, on the other hand, depends mainly on galaxy mass, and only weakly on environment.

left). The remarkable interdependencies of morphology, structure, mass, and environment are visually illustrated in Figure 4.

It is noteworthy that the dependence of galaxy structure on mass is much stronger than the morphological dependence on environment. Over 3 orders of magnitude in local surface density, early-type fractions change only from $\sim 25 \%$ to $\sim 50 \%$, whereas the fraction of galaxies with $n>2.5$ increases from $\sim 25 \%$ to $\sim 75 \%$ over little more than 1 order of magnitude in mass (see Fig. 3). In that respect environment only plays a secondary role in shaping the galaxy population.

It is intuitively clear that the key to understanding the difference between structure and morphology, which is solely due to the bumpiness parameter, is star formation activity. The definition of morphology as used in this Letter, i.e., a combination of $n$ and $B$, which is a measure of the deviation from a smooth profile, explicitly uses signs of star formation (spiral arms and starforming regions) to separate late-type from early-type galaxies. This was quantified earlier by Takamiya (1999), who showed that residuals from smooth profile fits correlate with $\mathrm{H} \alpha$ emission lines maps. Furthermore, Baldry et al. (2006) show that color, like morphology, depends on environment for galaxies with a given mass, trends that we also see in our sample.

In Figure 5 we explicitly show the relation between color and $B$. For low-mass galaxies, which have a large range in color, the fraction of smooth galaxies, those with $B<0.25$, increases toward redder colors. A notable deviation from this trend is that the reddest galaxies with $(u-g)_{0} \gtrsim 1.85$ are generally not smooth. Visual inspection of this small number $(5 \%)$ of very red galaxies shows that these are edge-on disk galaxies and irregular galaxies, such that their colors can be explained by high extinction.

Since star formation is generally confined to disks, color gradients, like bumpiness, are expected to trace morphology.

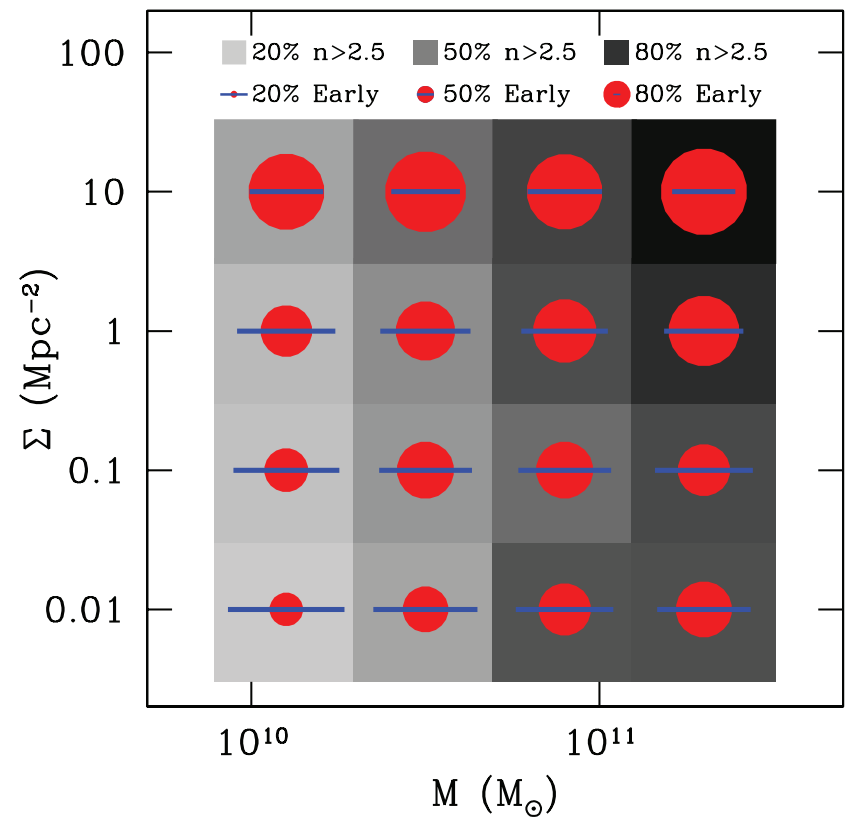

FIG. 4.-Graphical illustration of the result that morphology mainly depends on environment, and structure mainly on mass. The size of the filled, red circles (increasing from bottom to top) corresponds to the early-type galaxy fraction; the gray scale of the background (increasing from left to right) corresponds to the fraction of galaxies with $n>2.5$.

Park \& Choi (2005) design color versus color gradient criteria that, combined with concentration index, effectively distinguish visually classified early- and late-type galaxies. Following up on this, Park et al. (2007) find results similar to those we present here: they find that, at fixed luminosity, morphology depends on environment, whereas the dependence of the concentration index on environment is much weaker. It is encouraging that these results and ours are similar, because it is not self-evident that a morphological classification method based on colors behaves the same way as a method that involves spatial information that more directly corresponds to visual classifications.

We note that morphology behaves intrinsically differently as a function of stellar mass and luminosity. Park et al. (2007) find at fixed density a rather strong relation between luminosity and early-type galaxy fraction. At luminosities for which our

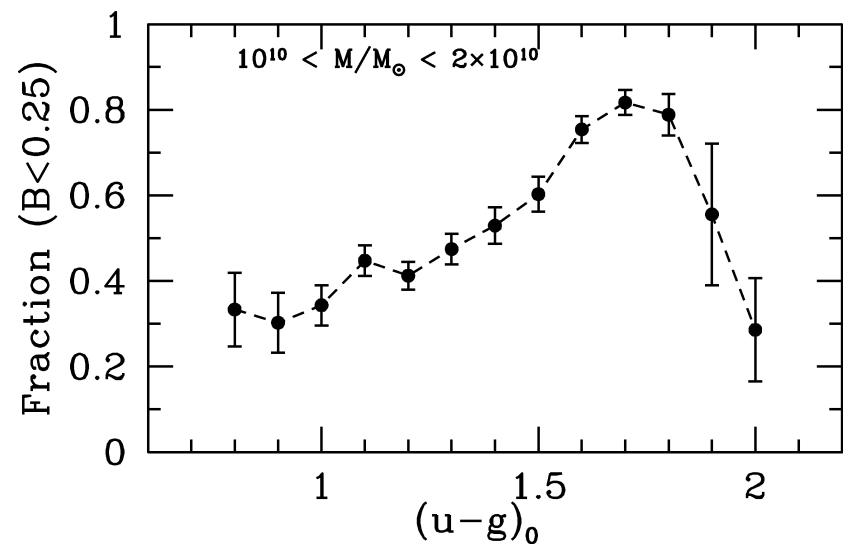

FIG. 5.-Fraction of smooth galaxies (with $B<0.25$ ) as a function of color for low-mass galaxies. The fraction of smooth, red galaxies is much larger than the fraction of smooth, blue galaxies (with the notable exception of the reddest galaxies). This implies that star formation is responsible for the increased bumpiness of blue galaxies. 
sample is complete, we also see an increased early-type fraction of high-luminosity galaxies. It is beyond the scope of this Letter to fully address this issue.

\section{CONSEQUENCES FOR HIGH-REDSHIFT STUDIES}

At higher redshifts $(z \sim 1)$ often some measure of galaxy structure is used to separate early-type from late-type galaxies (e.g., Abraham et al. 1996). Even more advanced measures of the light distribution than the concentration parameter $C$, such as $G$, the Gini coefficient, and $M_{20}$, the second-order moment of the brightest 20\% of the flux (Lotz et al. 2004; Capak et al. 2007; Zamojski et al. 2007), are essentially a measure of concentration for "normal" galaxies. $G$ and $M_{20}$ are very suitable to distinguish starbursting and merging galaxies, but for early-type and spiral galaxies the correlation between concentration and either of those parameters is too tight to effectively select the early types.

Even in classification methods which use asymmetry in addition to concentration (e.g., Schade et al. 1995), the problem sketched above is not necessarily remedied adequately: many late types, especially the massive ones, are rotationally symmetric, such that invoking asymmetry as a morphological classifier does not help much in distinguishing between $\mathrm{Sb}, \mathrm{Sa}$, and S0 galaxies (see, e.g., Conselice 2003). Only a parameter that quantifies the residual from a smooth light distribution can be successful in making that distinction. The bumpiness $B$ used in this Letter is one example of such a parameter; another one, the "clumpiness" parameter $S$, was introduced earlier by Conselice (2003), although Blakeslee et al. (2006) note that $S$ and $B$ are not interchangeable and that the combination of $n$ and $B$ seems to be more successful in separating early- and late-type galaxies.

Our conclusion that structure and morphology are intrinsically different galaxy properties has consequences for the interpretation of high-redshift morphological studies if those are based on some measure of structure. The obvious problem is that the fractions of highly concentrated and early-type galaxies are different, and that a direct comparison at different redshifts can lead to biases in the inferred evolution. A more subtle problem arises when the evolution in "morphological" properties is measured for galaxies with different masses or in a variety of environments. As we saw in $\S 3$, morphology and structure differ in their behavior as a function of mass and environment. Therefore, observed changes in, e.g., concentration are easily misinterpreted in terms of the effect of the environment or galaxy mass on the evolution of galaxies.

This type of problem can only be avoided if the methods used to classify low- and high-redshift galaxies are as similar as possible. This can be achieved by consistently using the same automated methods or by taking great care in using a fixed set of visual classification criteria for galaxies at different redshifts (as in, e.g., Postman et al. 2005).

\section{CONCLUSIONS}

We have shown that structure (in this Letter quantified by the Sérsic parameter $n$ ) and morphology (quantified by $n$ and the bumpiness parameter $B$ ) are distinct galaxy properties. There is a significant number of galaxies with high Sérsic indexes but late-type morphologies (Fig. 1). The physical significance of the difference between structure and morphology becomes apparent when their behavior as a function of galaxy mass and environment is analyzed. Structure mainly depends on galaxy mass whereas morphology, at fixed galaxy mass, depends on environment (Fig. 3). The different behavior is linked to star formation activity, which only weakly affects the structure of a galaxy, but strongly affects its morphological appearance (Fig. 5). This implies that the existence of the MDR is intrinsic, and is explained by the environmental dependence of star formation activity. This seems trivial; however, it means that the MDR is not simply the result of underlying correlations, i.e., the strong dependence of structure and star formation on galaxy mass, and the environmental dependence of the mass function.

The author gratefully acknowledges financial support from NASA grant NAG5-7697, and is indebted to support from John Blakeslee and Holland Ford. Stimulating discussions with Andrew Zirm and Marijn Franx helped shape this Letter. Attentive reading by and valuable comments from Andrew Zirm, Roderik Overzier, Alison Crocker, John Blakeslee, Bradford Holden, Ricardo Demarco, and Marc Postman have greatly improved the readability.

\section{REFERENCES}

Abraham, R. G., van den Bergh, S., Glazebrook, K., Ellis, R. S., Santiago, B. X., Surma, P., \& Griffiths, R. E. 1996, ApJS, 107, 1

Adelman-McCarthy, J. K., et al. 2007, ApJS, 172, 634

Baldry, I. K., Balogh, M. L., Bower, R. G., Glazebrook, K., Nichol, R. C., Bamford, S. P., \& Budavari, T. 2006, MNRAS, 373, 469

Bell, E. F., McIntosh, D. H., Katz, N., \& Weinberg, M. D. 2003, ApJS, 149, 289

Blakeslee, J. P., et al. 2006, ApJ, 644, 30

Brinchmann, J., Charlot, S., White, S. D. M., Tremonti, C., Kauffmann, G., Heckman, T., \& Brinkmann, J. 2004, MNRAS, 351, 1151

Capak, P. L., Abraham, R. G., Ellis, R. S., Mobasher, B., Scoville, N. Z., Sheth, K., \& Koekemoer, A. 2007, ApJS, 172, 284

Conselice, C. J. 2003, ApJS, 147, 1

Croton, D. J., et al. 2006, MNRAS, 365, 11

Dekel, A., \& Birnboim, Y. 2006, MNRAS, 368, 2

Diaferio, A., Kauffmann, G., Balogh, M. L., White, S. D. M., Schade, D., \& Ellingson, E. 2001, MNRAS, 323, 999

Dressler, A. 1980, ApJ, 236, 351

Farouki, R., \& Shapiro, S. L. 1981, ApJ, 243, 32

Gunn, J. E., \& Gott, J. R. 1972, ApJ, 176, 1

Hogg, D. W., et al. 2004, ApJ, 601, L29
Kauffmann, G., White, S. D. M., \& Guiderdoni, B. 1993, MNRAS, 264, 201

Kauffmann, G., et al. 2004, MNRAS, 353, 713

Larson, R. B., Tinsley, B. M., \& Caldwell, C. N. 1980, ApJ, 237, 692

Lotz, J. M., Primack, J., \& Madau, P. 2004, AJ, 128, 163

Moore, B., Katz, N., Lake, G., Dressler, A., \& Oemler, A. 1996, Nature, 379, 613

Park, C., \& Choi, Y.-Y. 2005, ApJ, 635, L29

Park, C., Choi, Y.-Y., Vogeley, M. S., Gott, J. R., \& Blanton, M. R. 2007, ApJ, 658, 898

Park, C., Gott, J. R., \& Choi, Y.-Y. 2008, ApJ, in press (arXiv:0708.4118)

Peng, C. Y., Ho, L. C., Impey, C. D., \& Rix, H.-W. 2002, AJ, 124, 266

Postman, M., et al. 2005, ApJ, 623, 721

Salpeter, E. E. 1955, ApJ, 121, 161

Schade, D., Lilly, S. J., Crampton, D., Hammer, F., Le Fevre, O., \& Tresse, L. 1995, ApJ, 451, L1

Sérsic, J. L. 1968, Atlas de Galaxias Australes (Cordoba: Observatorio Astronomico)

Takamiya, M. 1999, ApJS, 122, 109

van der Wel, A., et al. 2007, ApJ, 670, 206 (vdW07)

White, S. D. M., \& Frenk, C. S. 1991, ApJ, 379, 52

Zamojski, M. A., et al. 2007, ApJS, 172, 468 